\title{
Posterior approach to ventrally located spinal meningiomas
}

\author{
Spyridon Voulgaris $\cdot$ George A. Alexiou $\cdot$ Evaggelos Mihos $\cdot$ \\ Dimitrios Karagiorgiadis • Andreas Zigouris · George Fotakopoulos • \\ Dimitrios Drosos • Dimitrios Pahaturidis
}

Received: 28 May 2009/Revised: 11 November 2009/Accepted: 15 January 2010/Published online: 3 February 2010

(C) Springer-Verlag 2010

\begin{abstract}
For the resection of anteriorly located meningiomas, various approaches have been used. Posterior approach is less invasive and demanding; however, it has been associated with increased risk of spinal cord injury. We evaluated ten consecutive patients that underwent surgery for spinal meningiomas. All patients were preoperative assessed by neurological examination, computed tomography and magnetic resonance imaging. All tumors were ventrally located and removed via a posterior approach. Transcranial motor-evoked potentials (TcMEPs), somatosensory-evoked potential (SSEP) and free running electromyography (EMG) were monitored intraoperative. Postoperative all patients had regular follow-up examinations. There were four males and six females. The mean age was 68.2 years (range 39-82 years). In nine out of ten cases, the tumor was located in the thoracic spine. A case of a lumbar meningioma was recorded. The most common presenting symptom was motor and sensory deficits and unsteady gait, whereas no patient presented with paraplegia. All meningiomas were operated using a microsurgical technique via a posterior approach. During the operation, free running EMG monitoring prompted a surgical alert in case of irritation, whereas TcMEP and SSEP amplitudes remained unchanged. Histopathology revealed the presence of typical (World Health Organisation grade I) meningiomas. The mean Ki-67/MIB-1 index was $2.75 \%$ (range $0.5-7)$. None of our patients sustained a transient or permanent motor deficit. After a mean follow-up period of
\end{abstract}

S. Voulgaris · G. A. Alexiou $(\bowtie) \cdot$ E. Mihos ·

D. Karagiorgiadis - A. Zigouris · G. Fotakopoulos ·

D. Drosos - D. Pahaturidis

Department of Neurosurgery, University Hospital of Ioannina,

PO BOX 103, Neohoropoulo, 45500 Ioannina, Greece

e-mail: alexiougrg@yahoo.gr; me01929@cc.uoi.gr
26 months (range 56-16 months), no tumor recurrence and no instability were found. Posterior approach for anteriorly located meningiomas is a safe procedure with the use of intraoperative monitoring, less invasive and well-tolerated especially in older patients. Complete tumor excision can be performed with satisfactory results.

Keywords Meningiomas - Spine - Posterior approach · Intraoperative monitoring

\section{Introduction}

Spinal meningiomas constitute approximately $25 \%$ of all spinal tumors and are the second most common tumors after the nerve sheath. There is a female predominance and nearly $80 \%$ occur in the thoracic spine $[1,2]$. The majority are benign and classically found in an intradural extramedullary location. Spinal meningiomas are usually localized lateral to the spinal cord, whereas in $15-27 \%$ of cases are located anterior to the spinal cord and constitute a surgical challenge [3]. Herewith, we report on ten cases of anteriorly located spinal meningiomas that were totally excised via a posterior approach with the use of intraoperative monitoring.

\section{Materials and methods}

We evaluated ten consecutive patients that underwent surgery for spinal meningiomas between January 2005 and January 2009. All patients were preoperative assessed by neurological examination, computed tomography (CT) and magnetic resonance imaging (MRI) of the spine. All tumors were ventrally located and removed via a posterior approach. Transcranial motor-evoked potentials (TcMEPs), 
somatosensory-evoked potentials (SSEPs) and free running electromyography (EMG) were monitored intraoperative by an experienced neurophysiologist (DK). Postoperative all patients had regular follow-up examinations.

\section{Operative technique}

The patient is positioned prone. After careful preoperative planning, a midline skin incision is performed extending two levels above and below the extent of the lesion. The paraspinal, intrinsic dorsal and deep transversospinal muscles are separated subperiosteally on either side. Depending on tumor size, we performed a monosegmental or multisegmental laminectomy above and below the extent of the tumor. A partial facetectomy $(\sim 30 \%)$ is performed in order to increase the viewing angle. Pedicles removal was not performed in any case. The dura was opened in the midline and fixed to the sides. Dentate ligament section is usually required to facilitate spinal cord handling. The spinal cord is usually displaced dorsally and rotated by the tumor. Tumor removal begins laterally where the tumor attaches to the dura, in order to minimize thermal injury to the spinal cord. The meningioma shrinks and then is debulked in order to create more space. After tumor removal and careful hemostasis, the dura is coagulated in all cases and primarily closed in a watertight manner.

\section{Technique for TcMEP, SSEP and EMG}

Multipulse transcranial electrical stimulations were generated with an XLTEK stimulator. The electrodes were placed to $\mathrm{C} 1 / \mathrm{C} 2$ position of the International 10-20 system. Stimulus intensity was gradually increased from 0 to $200 \mathrm{~mA}$ and the duration from 0.2 to $0.5 \mathrm{~ms}$. The first recording was stored as baseline and was compared to the postoperative value. Regarding interpretation of the TcMEP, we used the total loss of amplitude as suggestive of damage. Free running EMG monitoring was performed during operation. The EMG electrodes were placed to the muscles (Netter's dermatomes) according to the thoracic and lumbar segment operated on, in order to record the corresponding nerve roots. Concerning meningiomas in the thoracic region, the electrodes were placed apart from the corresponding muscles (i.e. intercostals, forceps) to the muscles of the lumbar section.

Stimulation of SSEP was performed with electrical pulses of 300-s duration and a maximum intensity of $35 \mathrm{~mA}$ at a frequency of $4.7 \mathrm{~Hz}$. Stimulating electrodes were placed over each tibial nerve and the evoked potentials were recorded from the popliteal fossa (right and left),
C5S (cervical 5) or C2S, CP3 (central parietal) and CP4, $\mathrm{CPz}$ and $\mathrm{FPz}$ (frontal parietal), according to the International 10-20 system. Significant SSEP changes were defined as more than $50 \%$ reduction or over $10 \%$ delay from baseline values.

\section{Anesthesia}

Anesthesia was maintained with continuous propofol infusion $(20 \mathrm{ml} / \mathrm{h})$. No muscle relaxants were used after induction and intubation. Invasive blood pressure, ECG, end-tidal carbon dioxide concentration, pulse oximetry and temperature were monitored.

\section{Results}

Patient's data are summarized in Table 1. There were four males and six females. The mean age was 68.2 years (range 39-82 years). In nine out of ten cases, the tumor was located in the thoracic spine. A single case of a lumbar meningioma was recorded. All meningiomas were intradural and ventrally located to the spinal cord. The most common presenting symptom was motor and sensory deficits and unsteady gait, whereas no patient presented with paraplegia. All meningiomas were operated using a microsurgical technique via a posterior approach (Figs. 1, 2). During the operation, free running EMG monitoring prompted a surgical alert in case of irritation of the lumbar section recordings. In these cases, we ran TcMEP in order to identify changes in the amplitude. In all cases, no change of TcMEP was recorded suggesting no neurological deterioration (Fig. 3). SSEP was also unchanged. Histopathology revealed the presence of typical (World Health Organization grade I) meningiomas. Five meningiomas were psammomatous type, 3 transitional and 2 meningotheliomatous. The mean Ki-67/MIB-1 index, which is a proliferation index of the tumor, was $2.75 \%$ (range $0.5-7$ ). None of our patients sustained a transient or permanent motor deficit. Postoperative all patients had marked neurological improvement. After a mean follow-up period of 26 months (range 56-16 months), no tumor recurrence was noted on MRI evaluation and no instability.

\section{Discussion}

Spinal intradural extramedullary tumors account for $2 / 3$ of all intraspinal neoplasms and are mainly represented by meningiomas and schwannomas, with the former accounting for approximately $25 \%$ of all primary intraspinal tumors [1]. Spinal meningiomas occur less 
Table 1 Summary of patient's data

\begin{tabular}{|c|c|c|c|c|c|c|}
\hline & Age/sex & Location & Symptoms & Grade & Histology & MIB-1 (\%) \\
\hline 1 & $60 / \mathrm{F}$ & Thoracic & Radicular pain, sensory symptoms & I & Psammomatous & 1 \\
\hline 2 & $80 / \mathrm{M}$ & Thoracic & Unsteady gait, sphincter disturbances & I & Meningotheliomatous & 4 \\
\hline 3 & $56 / \mathrm{F}$ & Thoracic & Motor deficits & I & Psammomatous & 2 \\
\hline 4 & $70 / \mathrm{M}$ & Thoracic & Motor deficits, radicular pain & I & Transitional & 1 \\
\hline 5 & $63 / \mathrm{F}$ & Lumbar & Motor deficits & I & Psammomatous & 0.5 \\
\hline 6 & $39 / \mathrm{F}$ & Thoracic & Radicular pain & I & Transitional & 7 \\
\hline 7 & $57 / \mathrm{F}$ & Thoracic & Motor deficits & I & Psammomatous & 7 \\
\hline 8 & $79 / \mathrm{M}$ & Thoracic & Unsteady gait, motor deficits & I & Meningotheliomatous & 1 \\
\hline 9 & $73 / \mathrm{F}$ & Thoracic & Radicular pain & I & Psammomatous & $<0.5$ \\
\hline 10 & $85 / \mathrm{M}$ & Thoracic & Motor deficits & I & Transitional & 4 \\
\hline
\end{tabular}

Fig. 1 Illustrative case of patient no. 3, a 56-year-old female with an anterior located thoracic meningioma at T7-T8 level. The tumor displayed intermediate signal intensity on sagittal T2-weighted MRI (a), marked enhancement after intravenous contrast administration (b). c Gadolinium-enhanced T1-weighted MRI 1 year later without evidence of tumor recurrence
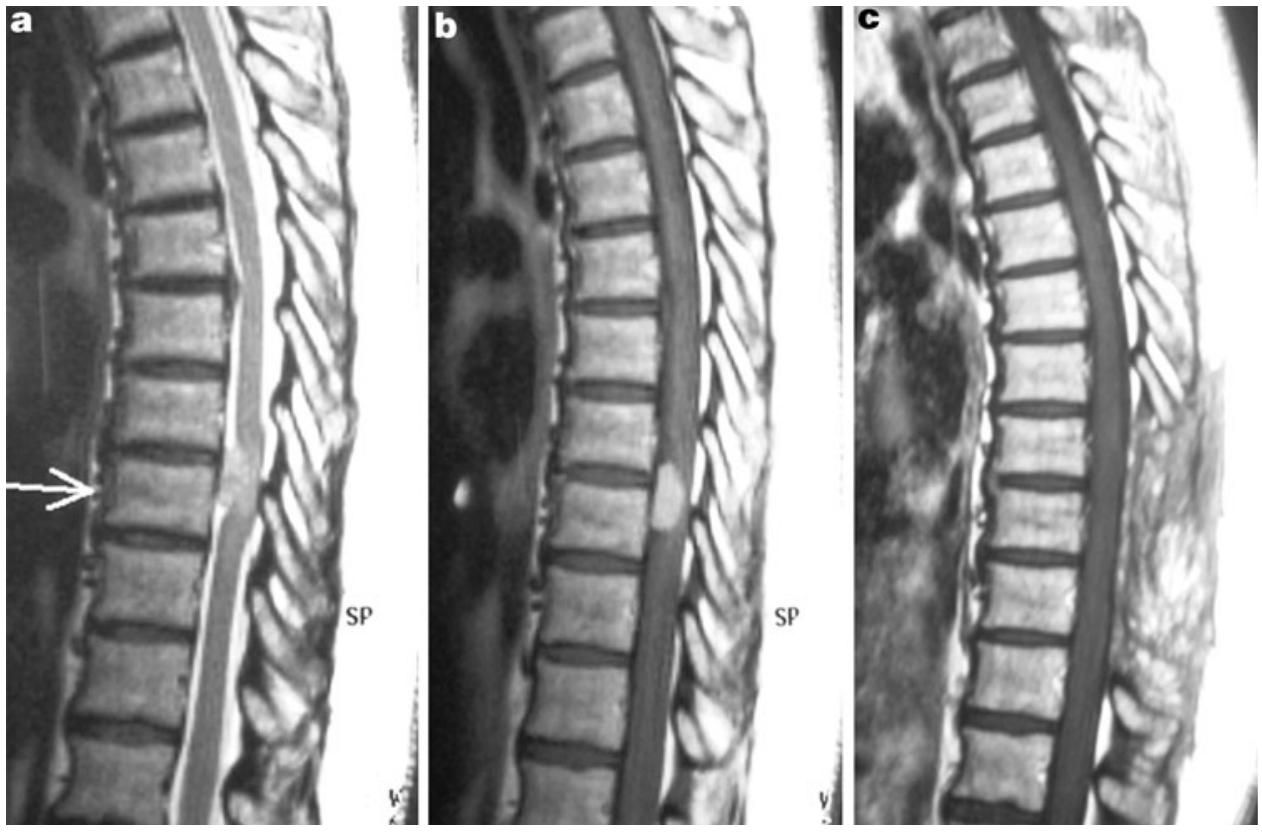

frequently than intracranial ones and account for approximately $7.5-12.7 \%$ of all meningiomas [2]. They are usually affecting middle-aged women and arise in the thoracic spine. Meningiomas also occur in the cervical and lumbar regions, albeit less frequently. Cohen-Gadol et al. [3] reported that patients younger than 50 years of age had more frequently meningiomas located in the cervical spine. Multiple tumors can occur only rarely. In the majority of cases, they are intradural like in our series. Epidural meningiomas account for only 3.5-7.0\% of all spinal meningiomas and occur more commonly in children than in adults $[4,5]$.

Symptomatology depends on tumor location and usually involves motor and sensory deficits, local or radicular pain, sphincter disturbances and paraplegia. Histopathologically spinal meningiomas are usually benign. Meningotheliomatous and psammomatous types are the most common, similar to our series. Ki-67 index has been found as an independent predictor of both tumor recurrence and overall survival in intracranial meningiomas [6]. Nevertheless, the value of Ki-67 index in spinal meningiomas has not been yet fully elucidated. Roser et al. compared spinal and intracranial meningiomas and reported that the proliferation rates of intracranial meningiomas were significant higher. The mean Ki-67 index was $2.48 \%$. Likewise, in our series, the mean Ki-67 index was $2.75 \%$ [7]. Nevertheless, further studies are needed to determine the Ki-67 role in predicting spinal meningiomas behavior. On MRI examination, spinal meningiomas have on T1-weighted images signal intensity similar to that of the spinal cord that does not substantial increased on T2-weighted images. Contrast enhancement is immediate and homogeneous. Lack of foraminal extension favors a diagnosis of meningioma over a diagnosis of schwannoma or neurofibroma [5]. 


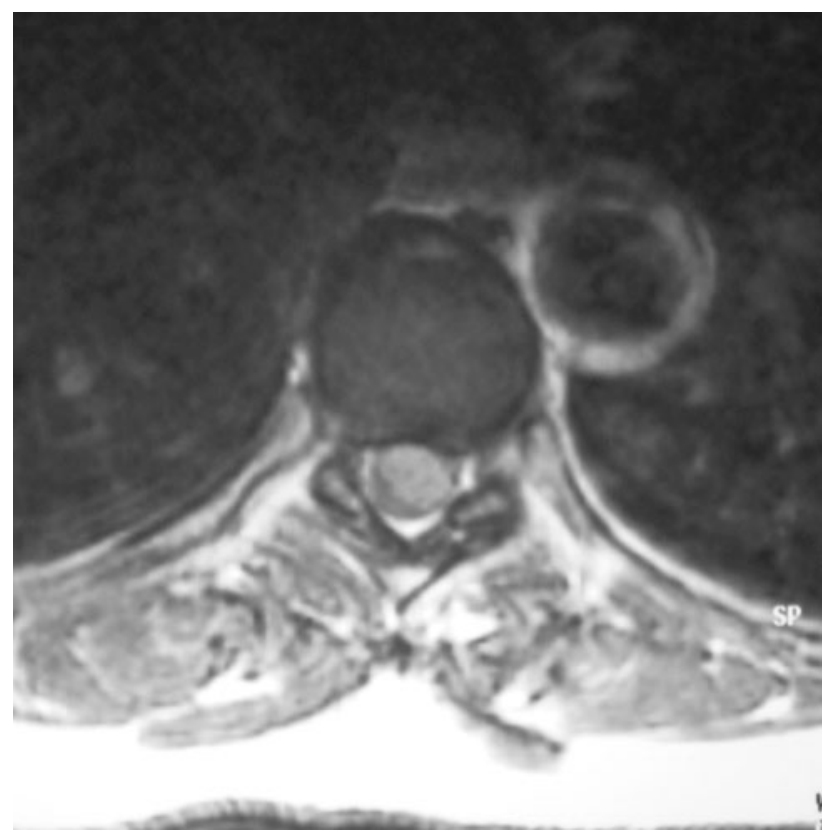

Fig. 2 Axial gadolinium-enhanced T1-weighted MRI revealing the thoracic meningioma and the dorso-lateral displacement of the spinal cord

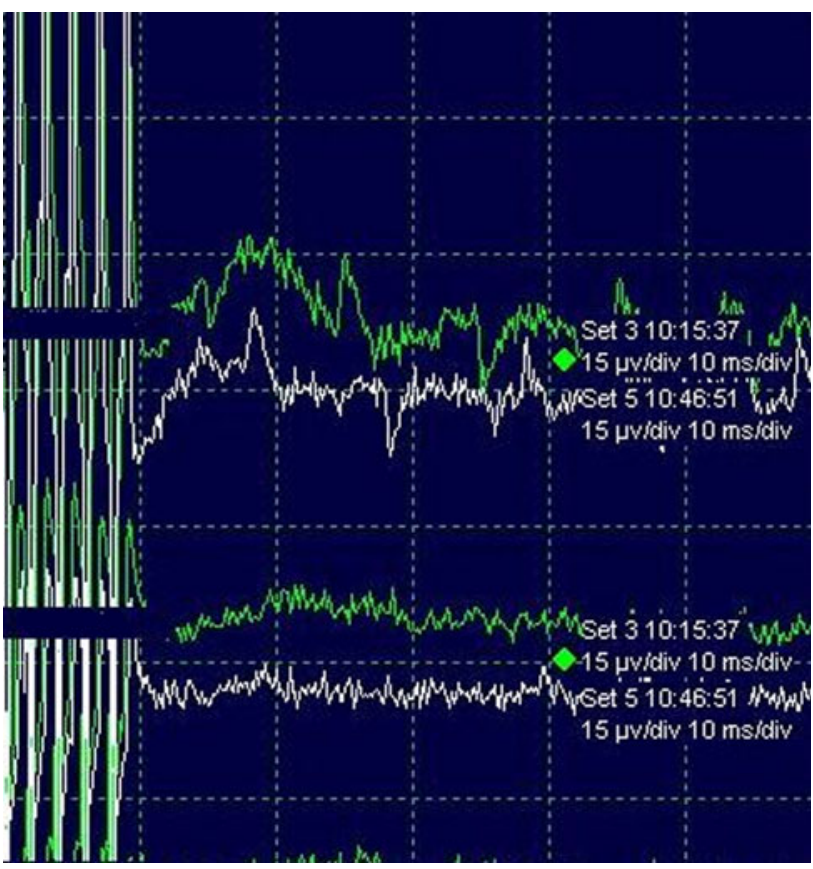

Fig. 3 Patient no. 3: Compared TcMEP values before (green wave) and after removal of a thoracic meningioma, revealing no significant amplitude change

Complete surgical excision is the treatment of choice. After total removal, significant neurological improvement is usually observed, whereas tumor recurrence rate has been reported to be $1.3-14 \%[2,8]$. Recently, radiosurgery has also been utilized for the treatment of intradural spinal tumors. Gerszten et al. [9] reported radiographic control of mainly recurrent meningiomas with radiosurgery; however, more studies are needed with longer follow-up periods. Advanced age does not seem to contraindicate surgery, even in patients with severe preoperative neurological deficits the quality of life can be improved in most cases [10]. For ventrally located spinal meningiomas, the transthoracic approach has been used. This approach provides direct access to the ventral surface of the spinal cord, enables dural manipulation and no spinal cord traction [11-13]. Nevertheless, a posterior approach is an alternative and offers fewer potential complications, eliminates the necessity of vertebrectomy, is less invasive and technically more easy [14-17]. The greatest disadvantage of this approach is the limited exposure, insufficient space to expose the disease and the need for spinal cord manipulation. If the operative field is narrow, scarification of one or more nerve roots has been suggested for widening the field to prevent spinal cord damage [8]. A postero-lateral approach has also been used with good results $[12,14]$. Gambardella et al. [12] reported radical excision of ten ventral thoracic meningiomas via a postero-lateral approach.

Damage of the spinal cord and incomplete resection of anterior spinal lesions via a midline posterior laminectomy has been reported $[2,14,18,19]$. In our series, we managed to safely excise all tumors by the use of TcMEP and continuous EMG. Intraoperative monitoring has been increasingly used as a method to prevent neurological injury during spine surgery. SSEPs and/or TcMEP have been applied for preventing motor deficits in spine surgery. Although SSEPs are easily recordable without adjusting the anesthetic regimen, there are reports of false negative results in up to $25 \%[15,19,20]$. TcMEPs have been used as an alternative with favorable results [21]. TcMEPs evaluate the pyramidal motor pathways and in combination with continuous EMG monitoring give an immediate and conclusive feedback of motor tract integrity. Furthermore, we found that TcMEPs may hold an additional prognostic role for predicting patient's postoperative improvement [22]. Nevertheless, the choice and management of the anesthetic agents may impair the diagnosis of intraoperative spinal cord injury [23]. TcMEPs are significantly affected by the use of halogenated agents and nitrous oxide as well as the level of neuromuscular blockade because these agents affect the neural synaptic and axonal functional activities [23, 24]. At an alveolar concentration (MAC) of 1.0 or higher, TcMEPs are often unobtainable. For that reason, a close working relationship of the monitoring team, the anesthesiologist, and the surgeon is key to the successful conduct and interpretation of TcMEP. In our series, TcMEP and EMG prompted alert in case of imminent surgical damage. 
Although the present study had a limited follow-up period of 26 months, the posterior approach with the use of intraoperative monitoring for anteriorly located meningiomas seemed to be a safe procedure, less invasive and welltolerated especially in older patients. Complete tumor excision can be performed with satisfactory results.

\section{References}

1. Albanese V, Platania N (2002) Spinal intradural extramedullary tumors. Personal experience. J Neurosurg Sci 46:18-24

2. Solero CL, Fornari M, Giombini S (1989) Spinal meningiomas: review of 174 operated cases. Neurosurgery 25:153-160

3. Cohen-Gadol AA, Zikel OM, Koch CA (2003) Spinal meningiomas in patients younger than 50 years of age: a 21 -year experience. J Neurosurg (Spine) 98:258-263

4. Weil SM, Gewirtz RJ, Tew JM (1990) Concurrent intradural and extradural meningioma of the cervical spine. Neurosurgery 27:629-631

5. Messori A, Rychlicki F, Salvolini U (2002) Spinal epidural en-plaque meningioma with an unusual pattern of calcification in a 14-year-old girl: case report and review of the literature. Neuroradiology 44:256-260

6. Bruna J, Brell M, Ferrer I, Gimenez-Bonafe P (2007) Ki-67 proliferative index predicts clinical outcome in patients with atypical or anaplastic meningioma. Neuropathology 27:114-120

7. Roser F, Nakamura M, Bellinzona M (2006) Proliferation potential of spinal meningiomas. Eur Spine J 15:211-215

8. Gottfried ON, Gluf W, Quinones-Hinojosa A (2003) Spinal meningiomas: surgical management and outcome. Neurosurg Focus 14:e2

9. Gerszten PC, Burton SA, Ozhasoglu C (2008) Radiosurgery for benign intradural spinal tumors. Neurosurgery 62:887-895

10. Sacko O, Haegelen C, Mendes V (2009) Spinal meningioma surgery in elderly patients with paraplegia or severe paraparesis: a multicenter study. Neurosurgery 64:503-509

11. D'Aliberti G, Talamonti G, Villa F (2008) Anterior approach to thoracic and lumbar spine lesions: results in 145 consecutive cases. J Neurosurg Spine 9:466-482
12. Gambardella G, Gervasio O, Zaccone C (2003) Approaches and surgical results in the treatment of ventral thoracic meningiomas. Review of our experience with a postero-lateral combined transpedicular-transarticular approach. Acta Neurochir (Wien) 145:385-392

13. Giroux JC, Nohra C (1978) Anterior approach for removal of a cervical intradural tumor: case report and technical note. Neurosurgery $2: 128-130$

14. Steck JC, Dietze DD, Fessler RG (1994) Posterolateral approach to intradural extramedullary thoracic tumors. J Neurosurg 81:202-205

15. Holland NR (1998) Subcortical strokes from intracranial aneurysm surgery: implications for intraoperative neuromonitoring. J Clin Neurophysiol 15:439-446

16. Gezen F, Kahraman S, Canakci Z (2000) Review of 36 cases of spinal cord meningioma. Spine 25:727-731

17. Levy W, Bay J, Dohn D (1982) Spinal cord meningioma. J Neurosurg 57:804-812

18. Jho HD (1997) Endoscopic microscopic transpedicular thoracic discectomy. Technical note. J Neurosurg 87:125-129

19. Krieger D, Adams HP, Albert F (1992) Pure motor hemiparesis with stable somatosensory evoked potential monitoring during aneurysm surgery: case report. Neurosurgery 31:145-150

20. Mizoi K, Yoshimoto T (1993) Permissible temporary occlusion time in aneurysm surgery as evaluated by evoked potential monitoring. Neurosurgery 33:434-440

21. Welch WC, Rose RD, Balzer JR (1997) Evaluation with evoked and spontaneous electromyography during lumbar instrumentation: a prospective study. J Neurosurg 87:397-402

22. Voulgaris S, Karagiorgiadis D, Alexiou G, Mihos E, Zigouris A, Fotakopoulos G, Drosos D, Pahaturidis D (2010) The value of transcranial motor evoked potentials and electromyography monitoring in spinal stenosis surgery. J Clin Neurosci. doi: 10.1016/j.jocn.2009.04.013

23. Sloan TB, Heyer EJ (2002) Anesthesia for intraoperative neurophysiologic monitoring of the spinal cord. J Clin Neurophysiol 19:430-443

24. Lo YL, Dan YF, Tan YE (2006) Intraoperative motor-evoked potential monitoring in scoliosis surgery: comparison of desflurane/nitrous oxide with propofol total intravenous anesthetic regimens. J Neurosurg Anesthesiol 18:211-214 